\title{
Agregometría plaquetaria, un abordaje sencillo de un estudio complejo
}

- María Alejandra Bravo Garzón, Gloria Ramos Ramos, Diego Galvis Roncancio, Barbarita María Mantilla Cardozo, Óscar Iván Reyes Cortés

Correo electrónico: gloriaramoshmc@gmail.com

Introducción: simplificar la interpretación de la agregometría plaquetaria en adultos con sospecha de trastornos de la función plaquetaria usando un panel de agonistas que incluye el ácido araquidónico.

Métodos: plasmas de sujetos sanos fueron analizados para obtener curvas de agregación plaquetaria con cuatro agonistas (ADP, epinefrina, colágeno y ácido araquidónico). Se obtuvieron los valores máximos de agregación para cada agonista. Los datos sobre el porcentaje máximo de agregación fueron analizados con el método de Shapiro-Wilk.

Resultados: 90 sujetos sanos contribuyeron con 270 muestras. Los porcentajes máximos de agregación por transmisión de luz para cada agonista no siguieron una distribución normal, por lo cual se expresaron como medianas: $85,7 \%, 86,1 \%, 84,3 \%$ y $88,4 \%$ para ADP $5 \mu \mathrm{M}$, colágeno $2 \mu \mathrm{g} / \mathrm{ml}$, epinefrina $10 \mu \mathrm{M}$ y ácido araquidónico $1 \mu \mathrm{M}$, respectivamente. Puesto que el percentil 2,5 fue mayor al $60 \%$ para todos los agonistas, se escogió este valor como el límite inferior de la normalidad.

Conclusión: el porcentaje máximo de agregación plaquetaria es el parámetro más importante para la evaluación de los resultados de las curvas de agregación plaquetaria, permitiendo simplificar la interpretación de la agregometría en adultos, incluyendo un agonista como el ácido araquidónico.

\section{Reacción leucemoide que simula una neoplasia mieloproliferativa crónica en mieloma múltiple indolente: reporte de caso y revisión de la literatura}

\section{Humberto Martínez, Jaime Valdés}

Correo electrónico: humbertomartinez48@hotmail.com

Introducción: la reacción leucemoide (RL) en el contexto de neoplasias de células plasmáticas se ha descrito en reportes de casos. No hemos encontrado asociación en la literatura de RL con mieloma indolente.

Métodos: se describe un caso de RL que simula una neoplasia mieloproliferativa crónica en mieloma múltiple indolente.

Resultados: mujer de 68 años con antecedente de diabetes mellitus tipo 2 y rosácea, con astenia, adinamia y malestar general. Dentro de los laboratorios inicialmente solicitados, se reporta una leucocitosis de $74.690 \times \mathrm{mm} 3$ con $67.190 \times \mathrm{mm} 3$ neutrófilos, sin anemia y recuento plaquetario normal. Se comienza tratamiento extrainstitucional con hidroxiurea y, posteriormente, con nilotinib. Se confirma la ausencia de cromosoma Filadelfia y mutación JAK2 V617F, por lo cual se suspende nilotinib y se documenta infiltración del $30 \%$ por neoplasia de células plasmáticas en médula ósea, y una paraproteinemia IgA lambda de 3,2 $\mathrm{g} / \mathrm{dl}$, sin componente CRAB por ausencia de anemia, alteración renal e hipercalcemia con PET/CT negativo para lesiones líticas o plasmocitomas, cadenas livianas relación menor de 100. Se considera mieloma múltiple indolente de alto riesgo por IgA y se inicia tratamiento con lenalidomida y dexametasona. La paciente se encuentra en aceptables condiciones generales, tolerando satisfactoriamente la quimioterapia.

Conclusión: este es el primer caso conocido por nosotros de mieloma múltiple indolente asociado con reacción leucemoide en la literatura mundial. 